\title{
A Detailed Research on Detection of Polycystic Ovary Syndrome from Ultrasound Images of Ovaries
}

\section{Gopalakrishnan, M. Iyapparaja}

\begin{abstract}
A common endocrine disorder named polycystic ovarian syndrome (PCOS) usually occurs to several women's during their reproductive age. This type of disease leads to infertility which represent with amenorrhea and hirsutism. The combination of clinical, endocrinological, and biochemical abnormalities are known to be PCOS which particularly related with the metabolism of estrogen and androgens. The periphery of ovaries is identified with the most common immature follicles which are less than 10mm. Analyzing this kind of situations in women is a major challenge which now doctors used ultrasound images which have the necessary details like number of follicles, size, and position. For real-time analysis of PCOS is a major task as follicles contains different sizes and highly connected with tissues and blood vessels which results in error prone. Several researchers have proposed different techniques for analyzing the PCOS using the ultrasound images of ovaries. In this study, we analyze the important factors and techniques used in detection of PCOS by using the ultrasound images taken from the women's ovary and compared the results of existing works.
\end{abstract}

Index Terms--- Polycystic Ovary Syndrome (PCOS), Ultrasound Image, Follicle Detection, Clustering Techniques

\section{INTRODUCTION}

The other terms for polycystic ovary syndrome (PCOS) is known as functional ovarian hyperandrogenism or SteinLeventhal syndrome which is a complex endocrine-disorder that are associated with the excess of androgens and longterm ovulation lacking. The ovaries several follicular cysts formation lead to characterize this PCOS disorder which known to be the process that ovum failed to release from the ovary [1]. The basic structure of a polycystic ovary is shown in figure 1. From the below figure, it has shown the polycystic ovary formed in women's reproductive system.

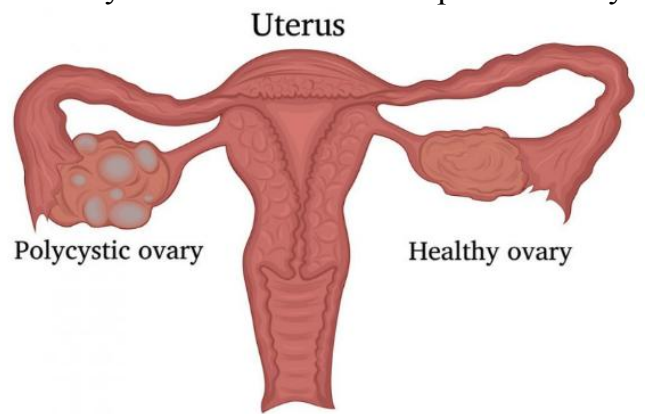

Fig. 1: Basic Diagram of Polycystic Ovary

The main features included in this reproductive system with PCOS are hyperandrogenaemia, chronic anovulation, enlarged ovaries with small follicles and insulin resistance. About 5 to $10 \%$ of women's are affected by PCOS who are

Manuscript Received September 16, 2019.

C. Gopalakrishnan, Research Scholar, SITE, Vellore Institute of

M. Iyapparaja, Associate Professor, SITE, Vellore Institute of Technology, Vellore. T.N, India. Technology, Vellore, T.N, India.

aged between 18 to 44 years old. Excess levels of insulin produced by insulin-resistance that causes elevated levels of blood-fats, male hormones excess production, and subsequent ovarian-dysfunction. The risk of diabetes, cardiovascular disease, obesity, heart diseases, and breast cancer is increased greatly when women found to have PCOSs. For this reason, it is much important to diagnose PCOS accurately. Figure 2 shows the polycystic ovaries of B-mode and Transvaginal-route [2].

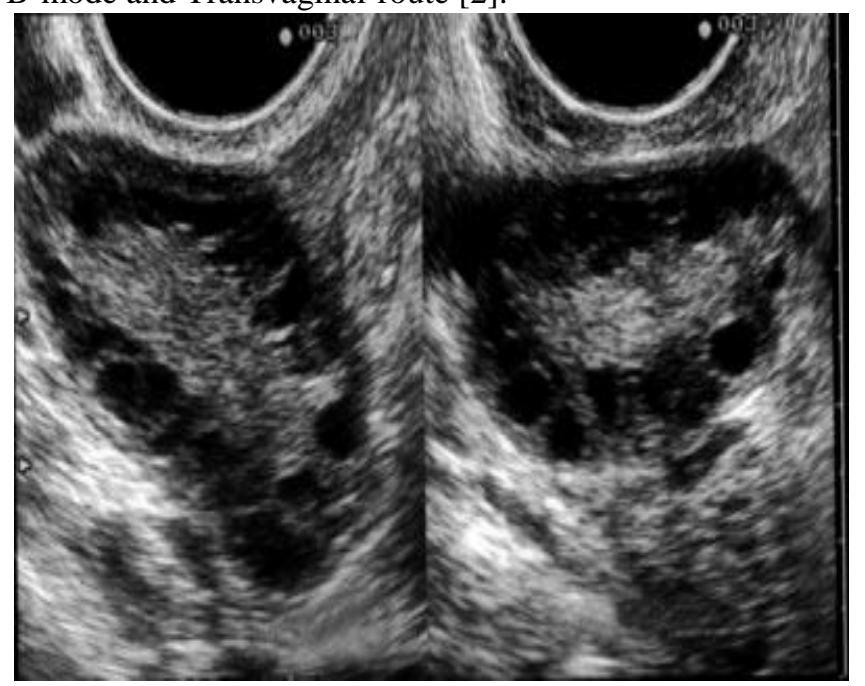

Fig.2: Images of Polycystic Ovaries of B-mode and Transvaginal Route

The above figure shows the images of B-mode polycystic ovary and transvaginal-route for the same. The width and length of the ovarian is increased with its area in the right and left ovaries.

\section{Polycystic Ovaries Histology and Appearance}

Morphology has made numerous descriptions on polycystic ovary which have refined time-to-time as imaging technology becomes advancement. During $18^{\text {th }}$ century, the first descriptions of pathology and anatomy have been suggested and features are made for the condition of polycystic ovary.

The ovary with fibrotic thickening, prominent theca of the multiple cystic follicles and tunica albuginea are the histology of the polycystic-ovary. The wedge resection is used as the only treatment for many years for PCOS and the routine practice is taken as histological assessment. The histological specimens are not readily available for polycystic ovaries as wedge resection is an outdated operation [3-5]. 
The observations of degenerating granulose cells and atretic follicles; luteinization and hypertrophy of the inner theca-cell layer; and thick ovarian tunica are defines the criteria of histopathologic. The studies examined the ovarian tissues that are obtained after wedge resection or hysterectomy which has shown the good correlation between the histopathological-criteria for polycystic ovaries and ultrasound-diagnosis of polycystic-morphology. The follicle number present in the polycystic ovary has 2 to 3 fold increase that indicated by the Hughesdons histological data. The cystic structures are identified as follicles which are opposed to pathological-cysts [6].

\section{ULTRASOUNDS TRANSABDOMINAL}

The diagnostic methods used commonly for polycystic ovary identification for the recent years are transvaginal or transabdominal ultrasound. The polycystic ovary diagnosis are not agreed universally when using ultrasound criteria, it is only accepted the characteristics features that are being increased in volume of stroma and ovary that is due to large number of follicles when comparing with normal ovaries [7]. The polycystic ovaries are described by the real-time high resolution ultrasound technique for one group with a static B-scanner that has $3.5 \mathrm{MHz}$ transabdominal. Unfortunately, it has been decided that the tiny follicles or cysts which cannot be detected by the ultrasound technique. The number of follicles which is having 2 to $6 \mathrm{~mm}$ diameter that is not defined or recorded also not describing stormal characteristics [8].

The static B-scanners limitations are hampered by the earlier studies but were suspended in the year 1980s for the high resolution which has sector scanners of real-time. The women's ovarian appearance is described by the ultrasound that classified as PCOS from the symptoms of serum endocrinology rather making the diagnosis [9]. Another group criterion for transabdominal ultrasound is attempted for defining polycystic ovary as a one that contains in single plane that represent atleast 10 follicles that are usually between 2 to $8 \mathrm{~mm}$ diameter arranged in the dense-core of ovarian storma peripherally. Several subsequent studies are adopted by the Adam's criterion that detects polycystic ovaries using ultrasound scanning. The diagnosis of symptoms and signs of women syndrome is supported by visualization of polycystic ovaries [10].

\section{ULTRASOUNDS TRANSVAGINAL}

Due to greater resolution, the transvaginal scanning is superior to that of transabdominal ultrasound for many cases that preferred for patients in which full bladder is avoided that saves time and comfortable. The infertility clinics context has the case of whilst in which repeated scans are done for women's that declined $20 \%$ of transvaginal for undergoing routine scanning after the diagnosis of transabdominal scan [11].

More accurately viewed the ovaries internal structure by using the transvaginal approach that avoids the homogeneous ovaries that are defined in the transabdominal scans for the patients with obese. The high frequency probe $>6 \mathrm{MHz}$ in transvaginal route that gives a better spatial resolution and examination depth is less which can be used as the vagina or the uterus is closer to the ovary and less disruptive of fatty tissues [12].

The NIH conference in the year 2012 confirms that the detection of PCOS by the Rotterdam criteria is the best and it is supported by the Endocrine Society. It is actually proposed in the year 2003 in which the volume of the ovary is more than $10 \mathrm{~cm}^{3}$ or equal. Even with these criteria, the doctors have to use ultrasound image for indicating the PCO.

\section{ULTRASONOGRAPHY IN PCOS}

The internal organs are monitored using the effective method known as ultrasonogrpahy (USG) that also includes the detection of women's infertility. The PCOS causes uterus problem which helps in determining infertility. The diagnosis of PCOS can be identified two of the three following criteria based on Rotterdam Consensus and these are amenorrhoea/oligo, follicles of PCO according to the result of USG, and biochemical or clinical symptom of excess activity of androgen [13].

Approximately, 2 to $15 \mathrm{Mhz}$ is used to diagnosis of PCO based on ultrasound which have another name as USG. The object is sent with the sound waves and reflected back in the form electrical-pulses. The display of processed pulses in the monitor is in the form of grayscale-image. Better accuracy is required for detecting the PCO follicles which have the high-noise characteristics and low contrast of USG images that are clustered and spherical. Two types of ovaries are presented in terms of morphological-features and these are polycystic and normal. Various sizes of mature follicles are grown by normal ovulation-phase. The $18 \mathrm{~mm}$ follicles are known as antral and those which are having 18 to $28 \mathrm{~mm}$ are referred as dominant. Therefore, 5 to 9 follicles are characterized as normal ovary that is sized between 2 to $28 \mathrm{~mm}$.

When the size of the follicles reaches 18 to $28 \mathrm{~mm}$ then it is mature enough to ovulate for polycystic ovary. In the ovulation-phase, the growing of PCO follicles is stopped when reaching the size between 5 to $7 \mathrm{~mm}$. The estrogen is produced again by the immature follicles which is a hormone that mucous membrane is regulated it growth in uterine-cavity. A thick mucous membrane is caused by the overproduced estrogen in a long period. Excessive bleeding or spotting occurs when blood circulation cannot accommodate by endometrium which is a symptoms due to PCOS [14].

\section{SEGMENTATION OF FOLLICLE \& RESULTS}

The oocytes are developed in ovarian follicles are fluid-filled structures and roughly spherical. The ultrasonogrphicpulses are not reflected for fluids of follicles images that are in two-dimensions which appeared as roughly, dark circular regions. An example of polycystic and normal ovary is shown in figure 3. A large number of possible and smaller irregular shaped follicles are exhibited by the PCO ovaries and also its distribution.

Published By:

Blue Eyes Intelligence Engineering

\& Sciences Publication 
Several techniques for follicle segmentation is proposed and categorized into graph-searching and grey-level thresholding techniques, texture-based methods, object recognition algorithms, and region growing methods [15].

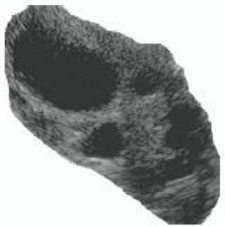

Normal Ovary

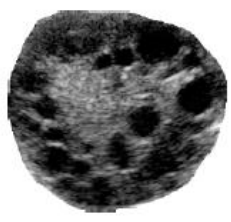

Polycystic Ovary
Fig. 3: Example of Normal-Ovary and Polycystic-Ovary

\section{IMMEDIATE TREATMENT FOR PCOS}

1. A total of $80 \%$ obese patients ovulation is restored by losing their body weight with atleast 5 to $7 \%$ that possibly reduce hyperinsulinaemia and thus elevation of BMI is used for hyperandrogenism.

2. Clomiphene citrate is the second step with induction of ovulation (OI) in this management which is limited with three-cycles. The insulin sensitizer is used for this management as a single-agent which more advisable.

3. Insulin sensitizing is followed by the next options like FSH hormone and Gonadotropin therapy. The ovulation is increased by the choices of drug named pharmacotherapy which includes Metformin that reduces the problem simultaneously that caused due to insulin resistance and excessive androgens of raised levels are regulated. The masculine effects like hirsutism and alopecia is reduced which is more advisable by anit-antorgenic therapy.

4. Surgical procedure are subjected further with the patients who are not having responsewith the Clomiphene therapy which named as Laproscopic Ovarian Drilling (LOD). Tissues produced by androgens are destroyed by this procedure and hormonal imbalance is corrected and restored the functioning of normal ovarian. The Testosterone levels and elevated LH are ultimately decreased and also FSH levels are increased.

\section{RELATED WORKS}

In general, detection of PCO follicles is having two applicable approaches and these are (1) Feature Extraction and Classification, and (2) Stereology. In the concept of stereology, the two-dimension images are perceived as estimation of three-dimensional objects. The parameters of three-dimensions are related with stereology which is obtained from the slice of 2D through structures. Stereology can be used to calculate the follicles geometric attributes like follicles-count, follicle distribution, and size of the follicle which is derived by MJ Lawrence et al. [16].

Stereology is used by B Purnama et al. [17] in his paper for measuring the diameter of follicle and for folliclequantification the Euclidean-distance is used. He proposed a method for detecting follicle by using the Euclideandistance and region growing a measured the size of the follicle with an accuracy level of $78 \%$. In his paper, only measurement and detection of follicle is undertaken.
Seatiawati et al. [18] used optimization technique named Particle Swarm Optimization for clustering ultrasound images by using a modified fitness function whish nonparametric. The extracted follicle features are classified by suggesting machine learning approaches.

Wisesty et al. [19] classified ultrasound images using three machine learning approaches and these are SVM, KNN using Eculideaen-distance, and NN-LVQ. Low pass filter, binarization, edge detection, morphology, and histogram average are used for preprocessing the ultrasound image. The Gabor Wavelet is used for manually extracting the features of the image which is used for classification. The results obtained by this technique is SVM has $82.55 \%$ and KNN with $78,81 \%$. N. SenthilMurugan and G. Usha Devi [20-23] have proposed hybrid techniques using machine learning approaches with optimization techniques and reviewed the concept and uses of other approaches and compared the results for their classification.

Dewailly et al. [24] compared the real-time counts of follicle using the proposed threshold 19-follicles per ovary for a large number of womens using the new ultrasound technology with $\mathrm{M}=62$ for PCOS and M=66 for controls. On average, comparison of 8 to 10 follicles per polycystic-ovary with the notation of follicles-count would be higher when the population uses the off-line approaches. The reproductive endocrinology-clinic having $M=105$ for the seeking the patients evaluation by applying the cluster analysis for excluding the population which considered for the PCOS functional evidence.

Akkasaligar and Malagavi[25] conducted a research for the PCOS ovary detection that contains 10 PCO-follicles. The polycystic characteristics are possessed by the cause of cyst-structured antral-follicles in numerous growths. The growth of polycystic ovary will be in numbers for antralfollicles but not in size which is a main difference and results in ovulation failure.

SiddharthSamsi et al. [26] detected follicles present in IHC slides by proposing an approach using the texture and color features for identifying regions of potential follicles. However, an ellipse fitting into the follicle will not be optimal due to its shape that varies significantly that depends on by its tissue. The number of centroblasts is depends on the Follicular Lymphoma grading in 10 randomly selected standard-microscopic high power fields (HPFs) that represents H\&E follicles stained tissue. The follicle regions are identified using the IHC stains in its tissue that later registered in $H \& E$ image that used for the diagnosisprocess. Moreover, excessive segmentation is resulted.

OclaySertelet al. [27] detected the centroblasts for an adaptive likelihood base cell-segmentation. The nuclear color for H\&E stain and the hues of blue for the cytoplasmic regions and for the collagen structures with protein rich has purple and hues of pink for the extracellular material. The 1$\mathrm{D}$ unitone image is converted from the input image using the principal component analysis (PCO). 
The construction of cellular-likelihood image is made by unitone values of the estimated parameter. The statistical measure is obtained by the matrix method of gray level runlength for the intensity variations of the spatial organization within each cell. Unique texture features are contained in the detected $\mathrm{CB}$ cells that clustered densely and non-CB will diverge from the dense-cluster.

Zhong Qu and Li Hang [28] presented the algorithm named 1D Otsu that employed widely due to its reliability and uncomplicated computation.

The gray-level pixel information is hardly evaluated by this 1D Otsu method instead of spatial neighborhood pixelinformation that results in poor segmentation. The function is ceased by this algorithm because of global distributions variation of the background and target. Good segmentation is provided by this method but having problem with the images having two unequal-classes. The author developed Entropy with hinged method to overcome the 1D Otsu techniques results.

W. X. Kang et al. [29] reviewed some important characteristics of image-segmentation algorithm by the ruling method. Otsu algorithm is classified as thresholding region based segmentation-algorithm.

Otsu thresholding-algorithms complexity rate is very high and deep slow for the processing rate. Better results are given by the segmentation algorithm and moreover it is needed to improve by integrating for better accuracy. $\mathrm{Z}$. Ningbo et al. [30] reduced the computation complexity of both $2 \mathrm{D}$ and $1 \mathrm{D}$ Otsu algorithms by presenting stable Otsualgorithm for an improved histogram.

The optimal threshold value is detected by applying the projection of 2D histogram in diagonal manner. The image is applied with Gaussian-noise, pepper and salt noise. The enhancement of threshold is improved by the results and immune for noise in the image but not for the Gaussian. L. Dongju and Y. Jian [31] described the K-means method that is similar with the Otsu technique for multilevel thresholding. The within class-variance is minimized which is the basic criteria for both models. Global thresholding is deployed by the Otsu technique and local-thresholding is employed by the K-means method.

Fang et al. [32] improved the enhancement result by proposing a technique with image-fusion method which has evaluation on sharpness.

The information perception for improving the image enhancement by C. Wang and Z. Ye [33] proposing a novel approach of extending the histogram equalization for the specification and to overcome the problem of Histogram Equalization (HE).

The essential idea of the proposed HE is entropy maximization as flat and Mary Kim and Min Gyo Chung [34] recursively weighted and separated the histogram equalization for contrast enhancement and brightness preservation.

Liu and $\mathrm{Li}$ [35] proposed the extension of Otsu method into $2 \mathrm{D}$ formation and threshold is switched into a vector. The segmentation result is improved greatly by this proposed method. Furthermore, the computation cost is increased significantly when optimal threshold is searched in 2 dimensional spaces that is the main limitation of this algorithm.

Huang and Wang [36] proposed Two Stage MultiThreshold Otsu technique that has put forwarded for the multi-level thresholding but the focus is still on gray-level images.

Han [37] used His color space of 2D-Otsu method for tree segmentation problem in color images. The background image would be simple and calculation of HIS color space for the trees $\mathrm{H}$ component is needed in advance that huge restriction could be applied.

K. Hammoudda et al. [38] proposed an unsupervised method with the help of K-means algorithm and Gabor filter for segmentation process. Three segmentation methods are presented and these are Expectation and Threshold maximization, K-means Clustering, and Thresholding due to the direct and clear nature with clustering approach that helps in categorizing the images efficiently.K. K. Singh and A. Singh [39] analyzed the various application areas using several image-segmentation techniques. The author discussed mainly Region based, Pixel based, and Edge based segmentation methods.

The color and seed region image-segmentation algorithms are discussed in this paper.

Cigale and Zazula [40] utilized cellular neural networks and cellular-automata for follicle segmentation. The results obtained from this technique are very promising but the major drawback of these methods is determinations are difficult for the required parameters of folliclesegmentation. Sarty et al. [41] analyzed the outer follicle wall-segmentation using the semi-automated method in which inner border manual tracing is accomplished using watershed-segmentation technique. The smooth image data is applied in watershed technique in which adjunct small follicles are merged. The areas are separated by applying the binary mathematical morphology.

P. S. Hiremath and J. R. Tegnoor [42] analyzed the infertility treatment with effective apparatus for ovarian ultrasound images.

The authors also analyzed the detection of follicle and employed the watershed-segmentation method. Poor segmentation is occurred due to speckle noise which difficult to find boundaries.

T. Chen et al. [43] presented a structural framework for the detection of follicle quantification in ultrasound $3 \mathrm{D}$ images.

The location and size of follicles of each ovarian is identified by the proposed system by the combination of data from the local and global context.

The strategy of database guided-segmentation is used for partitioning the candidates of follicles at detected locations.A learning approach named cluster outer space is used for searching the high-dimension space theory of multiple objects efficiently. 
Table 1: Overview of Literature Survey

\begin{tabular}{|c|c|c|c|}
\hline $\begin{array}{l}\text { S. } \\
\text { No. }\end{array}$ & Authors \& Year & Methodology & Limitations \\
\hline 1 & B. Bhanu et al. (1995) [44] & Genetic Algorithm (GA) is used & $\begin{array}{l}\text { Computational requirements are varied by } \\
\text { the number of parameters }\end{array}$ \\
\hline 2 & $\begin{array}{l}\text { T. Chiang and Y. Q. Zhang } \\
\text { (1997) [45] }\end{array}$ & Quadratic-Rate distortion method & Detection of minimum is failed \\
\hline 3 & R. Dass et al. (2012) [46] & $\begin{array}{l}\text { Artificial Neural Network, Thresholding } \\
\text { Method, Edge Based Method }\end{array}$ & $\begin{array}{l}\text { Higher level process is not allowed for } \\
\text { extracting all information }\end{array}$ \\
\hline 4 & N. Sharma et al. (2012) [47] & $\begin{array}{l}\text { Image processing and analysis, Normalized } \\
\text { cuts }\end{array}$ & Performance differentiation is difficult \\
\hline 5 & $\begin{array}{l}\text { S. Dhanalakshmi and T. } \\
\text { Ravichandran (2012) [48] }\end{array}$ & Circular partitioning, Quad Tree Decay & Method used is bottleneck \\
\hline 6 & $\begin{array}{l}\text { G. K. Seerha and R. Kaur (2013) } \\
\text { [49] }\end{array}$ & Dynamic Region-Merging & Homogeneity is placed \\
\hline 7 & H.P. Narkede (2013) [50] & $\begin{array}{l}\text { Threshold Method, Segmentation of image } \\
\text { on video }\end{array}$ & Computer vision problem \\
\hline 8 & $\begin{array}{l}\text { R. Khandelwal and Sanjay } \\
\text { Bhargav (2014) [51] }\end{array}$ & MR images and Demonizing & Intensity, Color, and Noise affects \\
\hline
\end{tabular}

\section{CONCLUSION}

In this paper, the major aspects of detecting PCOS from ultrasound images of ovaries is discussed and analyzed the existing works that are presented for the detection. Here, the basic concept of PCOS is studied with the histology and appearance. Several techniques were broadly categorized with the implantation based on humans and machine. Since, several techniques having own advantages in this application, comparison for a particular specifications are not given. Moreover, it is difficult to implement the process of detecting PCOS from ultrasound image ovaries in accurate manner. For this reason, several algorithms and techniques were proposed for the detection and classification of the PCOS. Many hybrid techniques also proposed to overcome the problem of detection but still the convenient method is to accomplish. For future scope, new hybrid techniques have to develop for improving the detection of PCOS from ultrasound images of ovaries.

\section{REFERENCES}

1. Özay, A. C., EmekçiÖzay, Ö., Okyay, R. E., \&Gülekli, B. (2019) The effect of myoinositol on ovarian blood flows in women with polycystic ovary syndrome. Gynecological Endocrinology, 1-5.

2. Ramamoorthy, S., Vinodhini, R., \&Sivasubramaniam, R. (2019, January). Monitoring the growth of Polycystic Ovary Syndrome using Mono-modal Image Registration Technique: Application of Medical Big Data in Healthcare. In Proceedings of the ACM India Joint International Conference on Data Science and Management of Data (pp. 180-187). ACM.

3. Katsigianni, M., Karageorgiou, V., Lambrinoudaki, I., \&Siristatidis, C. (2019). Maternal polycystic ovarian syndrome in autism spectrum disorder: a systematic review and meta-analysis. Molecular psychiatry, 1 .

4. Iyapparaja M, Tiwari. M, Security policy speculation of user uploaded images on content sharing sites, IOP Conf. Series: Materials Science and Engineering 263 (2017) 042018 doi:10.1088/1757899X/263/4/042019,pp-1-8.

5. M. Iyapparaja, P. Sivakumar. Metrics Based Evaluation for Disease Affection in Distinct Cities. Research J. Pharm. and Tech. vol. 10, no. 8, pp. 2487-2491,2017.

6. Rackow, B. W., Brink, H. V., Hammers, L., Flannery, C. A., Lujan, M. E., \&Burgert, T. S. (2018). Ovarian Morphology by Transabdominal Ultrasound Correlates With Reproductive and Metabolic Disturbance in Adolescents With PCOS. Journal of Adolescent Health, 62(3), 288-293.
7. Miao, Y., Wen, J., Huang, L., Wu, J., \& Zhao, Z. (2018). Diagnosis and Management of Ovarian Tumor in Mayer-Rokitansky-KüsterHauser (MRKH) Syndrome. BioMed research international, 2018.

8. Fulghesu, A. M., Porru, C., \&Canu, E. (2018). Diagnosis of Polycystic Ovarian Syndrome in Adolescence. In Good Practice in Pediatric and Adolescent Gynecology (pp. 143-159). Springer, Cham.

9. Thomas, N., \&Kavitha, A. (2018). A LITERATURE INSPECTION ON POLYCYSTIC OVARIAN MORPHOLOGY IN WOMEN USING DATA MINING METHODOLOGIES. International Journal of Advanced Research in Computer Science, 9(1).

10. Lee, H. J., Bahr, J. M., Bitterman, P., Basu, S., Sharma, S., Abramowicz, J. S., \&Barua, A. (2018). Polycystic Ovarian Condition May Be a Risk Factor for Ovarian Tumor Development in the Laying Hen Model of Spontaneous Ovarian Cancer. Journal of immunology research, 2018

11. Froyman, W., Van Schoubroeck, D., \& Timmerman, D. (2018). Automated follicle count using three- dimensional ultrasound in polycystic ovarian morphology. Ultrasound in Obstetrics \&Gynecology, 51(1), 147-149.

12. Katsigianni, M., Karageorgiou, V., Lambrinoudaki, I., \&Siristatidis, C. (2019). Maternal polycystic ovarian syndrome in autism spectrum disorder: a systematic review and meta-analysis. Molecular psychiatry, 1.

13. Feng, Y., Tamadon, A., \& Hsueh, A. J. (2018). Imaging the ovary. Reproductive biomedicine online, 36(5), 584-593.

14. Kumar, K. S., Nirmala, V., Venkatalakshmi, K., \&Karthikeyan, K. (2018). Analysis of Optimization Algorithms on Follicles Segmentation to Support Polycystic Ovarian Syndrome Detection. Journal of Computational and Theoretical Nanoscience, 15(1), 380-391.

15. Panchal, S., \&Nagori, C. B. (2018). CHAPTER Baseline Scan and Ultrasound 24 Diagnosis of PCOS. Donald School Textbook of Transvaginal Sonography, 250

16. Lawrence, M. J., Eramian, M. G., Pierson, R. A., \& Neufeld, E. (2007, May). Computer assisted detection of polycystic ovary morphology in ultrasound images. In Fourth Canadian Conference on Computer and Robot Vision (CRV'07) (pp. 105-112). IEEE.

17. Purnama, B., Hasyim, A., Septiani, M. D., Wisesty, U. N., \&Astuti, W. (2015). Follicle Detection on the USG Images to Support Determination of Polycystic Ovary Syndrome. In Journal of Physics: Conference Series (Vol. 622, No. 1, p. 012027). IOP Publishing.

18. Setiawati, E., \&Tjokorda, A. B. W. (2015, May). Particle swarm optimization on follicles segmentation to support PCOS detection. In 2015 3rd International Conference on Information and Communication Technology (ICoICT) (pp. 369-374). IEEE.

19. Wisesty, U. N., \&Nasri, J. (2016, August). Modified Backpropagation Algorithm for Polycystic Ovary Syndrome Detection Based on Ultrasound Images. In International Conference on Soft Computing and Data Mining (pp. 141-151). Springer, Cham.

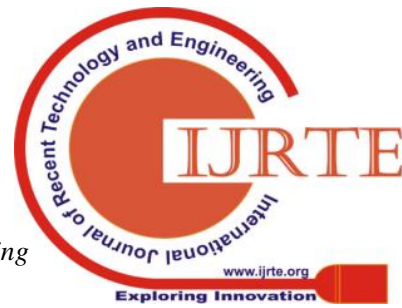


20. Nagarajan, S. M., \& Gandhi, U. D. (2018). Classifying streaming of Twitter data based on sentiment analysis using hybridization. Neural Computing and Applications, 1-9.

21. Iyapparaja M, Tiwari. M, Security policy speculation of user uploaded images on content sharing sites, IOP Conf. Series: Materials Science and Engineering 263 (2017) 042018 doi:10.1088/1757-899X/263/4/042019,pp-1-8

22. Murugan, N. S., \& Devi, G. U. (2018). Feature extraction using LRPCA hybridization on twitter data and classification accuracy using machine learning algorithms. Cluster Computing, 1-10.

23. Iyapparaja M, Bhanupriya Sharma, Augmenting SCA project management and automation Framework, IOP Conf. Series: Materials Science and Engineering 263 (2017) 042018 doi:10.1088/1757-899X/263/4/042018,pp-1-8

24. Dewailly, D., Gronier, H., Poncelet, E., Robin, G., Leroy, M., Pigny, P., \&Catteau-Jonard, S. (2011). Diagnosis of polycystic ovary syndrome (PCOS): revisiting the threshold values of follicle count on ultrasound and of the serum AMH level for the definition of polycystic ovaries. Human reproduction, 26(11), 3123-3129.

25. Akkasaligar, P. T., \&Malagavi, G. V. (2014, May). Detection of cysts in medical ultrasound images of ovary. In Proceedings of 5th SARCIRF International Conference (Vol. 4, pp. 57-62).

26. Samsi, S., Lozanski, G., Shanarah, A., Krishanmurthy, A. K., \&Gurcan, M. N. (2010). Detection of follicles from IHC-stained slides of follicular lymphoma using iterative watershed. IEEE Transactions on Biomedical Engineering, 57(10), 2609-2612.

27. Sertel, O., Lozanski, G., Shana'ah, A., \&Gurcan, M. N. (2010). Computer-aided detection of centroblasts for follicular lymphoma grading using adaptive likelihood-based cell segmentation. IEEE Transactions on Biomedical Engineering, 57(10), 2613-2616.

28. Qu, Z., \& Zhang, L. (2010, August). Research on image segmentation based on the improved Otsu algorithm. In 2010 Second International Conference on Intelligent Human-Machine Systems and Cybernetics (Vol. 2, pp. 228-231). IEEE.

29. Kang, W. X., Yang, Q. Q., \& Liang, R. P. (2009, March). The comparative research on image segmentation algorithms. In 2009 First International Workshop on Education Technology and Computer Science (Vol. 2, pp. 703-707). IEEE.

30. Zhu, N., Wang, G., Yang, G., \& Dai, W. (2009, November). A fast 2d otsu thresholding algorithm based on improved histogram. In 2009 Chinese Conference on Pattern Recognition (pp. 1-5). IEEE.

31. Liu, D., \& Yu, J. (2009, August). Otsu method and K-means. In 2009 Ninth International Conference on Hybrid Intelligent Systems (Vol. 1, pp. 344-349). IEEE.

32. Fang, X., Liu, J., Gu, W., \& Tang, Y. (2011, July). A method to improve the image enhancement result based on image fusion. In 2011 International Conference on Multimedia Technology (pp. 5558). IEEE.

33. Wang, Y., Chen, Q., \& Zhang, B. (1999). Image enhancement based on equal area dualistic sub-image histogram equalization method. IEEE Transactions on Consumer Electronics, 45(1), 68-75.

34. Kim, M., \& Chung, M. G. (2008). Recursively separated and weighted histogram equalization for brightness preservation and contrast enhancement. IEEE Transactions on Consumer Electronics, 54(3), 1389-1397.

35. Liu, J., Li, W., \& Tian, Y. (1993). The automatic thresholding of gray-level picture via 2D Otsu algorithm. ActaAutom. Sin, 19(1), 101-105.

36. Huang, D. Y., \& Wang, C. H. (2009). Optimal multi-level thresholding using a two-stage Otsu optimization approach. Pattern Recognition Letters, 30(3), 275-284.

37. Han, D., \& Huang, X. (2010, September). A tree image segmentation method based on 2-D OTSU in HSI color space. In 2010 6th International Conference on Wireless Communications Networking and Mobile Computing (WiCOM)(pp. 1-4). IEEE.

38. K. Hammouda, E. Jernigan, " Texture Segmentation using Gabor filter," University of Waterloo, Ontario, Canada, 2001.

39. Singh, K. K., \& Singh, A. (2010). A study of image segmentation algorithms for different types of images. International Journal of Computer Science Issues (IJCSI), 7(5), 414

40. Cigale, B., \&Zazula, D. (2004). Segmentation of ovarian ultrasound images using cellular neural networks. International Journal of Pattern Recognition and Artificial Intelligence, 18(04), 563-581.

41. Sarty, G. E., Liang, W., Sonka, M., \& Pierson, R. A. (1998). Semiautomated segmentation of ovarian follicular ultrasound images using a knowledge-based algorithm. Ultrasound in medicine \& biology, 24(1), 27-42

42. Hiremath, P. S., \&Tegnoor, J. R. (2010). Automatic detection of follicles in ultrasound images of ovaries using edge based method. IJCA, Special Issue on RTIPPR, 2, 120-125.
43. Chen, T., Zhang, W., Good, S., Zhou, K. S., \&Comaniciu, D. (2009, September). Automatic ovarian follicle quantification from $3 \mathrm{~d}$ ultrasound data using global/local context with database guided segmentation. In 2009 IEEE 12th International Conference on Computer Vision (pp. 795-802). IEEE.

44. Bhanu, B., Lee, S., \& Ming, J. (1995). Adaptive image segmentation using a genetic algorithm. IEEE Transactions on systems, man, and cybernetics, 25(12), 1543-1567.

45. Chiang, T., \& Zhang, Y. Q. (1997). A new rate control scheme using quadratic rate distortion model. IEEE Transactions on Circuits and Systems for Video Technology, 7(1), 246-250.

46. R.Dass, S. Devi, Priyanka, "Image Segmentation Techniques", International Journal Of Electronics And

Communication Technology, ISSN: 2230-9543, Vol.3, Issue 1, pp. 66-70, 2012

47. Sharma, N., Mishra, M., \&Shrivastava, M. (2012). Colour image segmentation techniques and issues: an approach. International Journal of Scientific \& Technology Research, 1(4), 9-12.

48. S. Dhanalakshmi, Dr. T. Ravichandran, "A New Method for Image Segmentation", IJARCSE, ISSN: 2277 128X, Vol. 2, Issue 9, pp. 293 299, Sep 2012

49. Seerha, G. K., \& Kaur, R. (2013). Review on recent image segmentation techniques. International Journal on Computer Science and Engineering, 5(2), 109

50. Narkhede, H. P. (2013). Review of image segmentation techniques. International Journal of Science and Modern Engineering, 1(8), 54-61.

51. R. Khandelwal, A. Kumar, S. Barghava, "Review Existing Image Segmentation Techniques" International Journal of Advanced Research in Computer Science and Software Engineering, ISSN: 2277 128X, Vol. 4, Issue 4, pp. 153-156, 2014

\section{AUTHORS PROFILE}

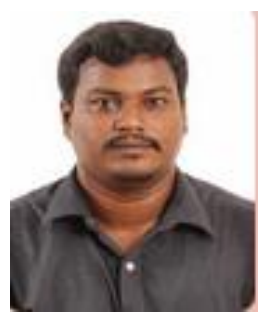

Gopalakrishnan, $\mathbf{C}$, He received his UG and PG degree in Computer science and Engineering discipline. He is doing his research in the area of machine learning in Health care domain. $\mathrm{He}$ published number of papers in referred impact factor journals. He registered his research work for the recognition of patent. arungopalit@gmail.com.

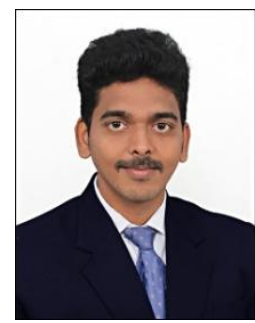

Iyapparaja Meenakshisundaram, $\mathrm{He}$ received Ph.D in Anna University, Chennai, BE degree from Anna University, Chennai and ME degree from Anna University of Technology, Coimbatore. Presently, $\mathrm{He}$ is an Associate Professor in School of Information Technology and Engineering, VIT, Vellore. He has 11 years of experience in Teaching and Big data, Software testing and software Engineering field. He received University Rank holder award for his ME degree. His research interests include Software Testing, Software Engineering, Big data, Networking and Agile Testing. He is life time member of ISTE.iyapparaja.m@vit.ac.in. 